\title{
Impact of the COVID-19 pandemic on trauma activations at a pediatric level 1 trauma center in New York
}

\author{
Yan Shi ${ }^{1,2} \cdot$ Charlotte Kvasnovsky ${ }^{1,2} \cdot$ Saira Khan $^{3} \cdot$ Shelley Jain $^{3,4} \cdot$ Danielle Sargeant $^{5} \cdot$ Abdulraouf Lamoshi $^{1,2}$. \\ Jose Prince $^{1,2} \cdot$ Chethan Sathya ${ }^{1,2}$
}

Accepted: 30 June 2021 / Published online: 15 July 2021

(c) The Author(s), under exclusive licence to Springer-Verlag GmbH Germany, part of Springer Nature 2021

\begin{abstract}
Background The disruptive effects on society and medical systems due to the coronavirus disease 2019 (COVID-19) pandemic are substantial and far-reaching. The effect of the pandemic on the quantity and quality of pediatric traumas is unclear and has a direct bearing on how scarce hospital resources should be allocated in a pandemic situation.

Methods A retrospective review of the trauma registry was performed for trauma activations in the years 2018 through 2020 during the months of March, April, and May. Demographic and injury specific datapoints were compared across calendar years.

Results There were 111, 100, and 52 trauma activations during the study interval in 2018, 2019, and 2020, respectively. There were fewer highest severity level activations in 2020 compared to 2018 and 2019 ( 1 vs 5 and $9 ; p<0.01$ ). The median Injury Severity Score was 5 in 2020 compared to 4 in both 2018 and $2019(p<0.01)$. More patients went directly to the operating room in 2020 compared to prior years $(21.2 \%$ vs $8 \%$ and $6.1 \% ; p<0.01)$. There were fewer discharges from the emergency department (ED) (12.1\% vs $36.6 \%$ and $32.7 \%)$. No increase in the number of child abuse reports and investigations was noted. There was no difference in the proportion of blunt versus penetrating trauma between years $(p=0.57)$. No pedestrians were struck by automobiles in 2020 compared to 12 and 14 in 2018 and 2019. However, there were a greater proportion of injuries from falls during 2020 compared to prior years.

Conclusions There were fewer trauma activations during the peak of the COVID pandemic compared to prior years. Due to the decrease in trauma volume during the peak of the pandemic, hospital resources could potentially be reallocated toward areas of greater need.
\end{abstract}

Level of evidence IV; Retrospective cohort study using historical controls.

Keywords COVID $\cdot$ Trauma $\cdot$ Pediatrics

\section{Introduction}

Since the emergence of the coronavirus disease 2019 (COVID-19) caused by the severe acute respiratory syndrome coronavirus 2 (SARS-CoV-2) in December of 2019, it has gone on to infect over 24 million people and kill over 820,000 around the globe as of August 2020 [1, 2]. Attempts to mitigate COVID-19 spread forced many hospitals to make

This paper will be presented as an ePoster at the 2020 Pediatric Trauma Society Annual Meeting. This article has not been submitted or presented elsewhere.

Yan Shi

yan.shi@uhhospitals.org

Extended author information available on the last page of the article dramatic changes in the way many non-COVID disease processes are evaluated and treated [3-5].

Cohen Children's Medical Center is the only Level 1 trauma center serving eastern New York City and Nassau County. We typically see 900 trauma patients with 180 trauma activations per year. On March 22nd, as cases were escalating in New York state, the state government initiated "NYS on PAUSE" which shutdown all non-essential businesses and limited travel and gatherings outside of the home. New trauma protocols were established within our trauma center to try to limit the unnecessary exposure of healthcare workers and conserve personal protective equipment (PPE) during trauma activations. Most notably, the mechanism in which traumas were activated was modified. 
There is limited data in the literature regarding the effect of the pandemic on pediatric trauma system volumes and injury mechanisms. We hypothesized that trauma volumes, especially motor vehicle-related traumas would diminish due to decreased traffic, fewer outdoor activities, and the possibility of delayed presentations due to reluctance to present to the hospital. We also expected a corresponding change in injury patterns and mechanisms due to the aforementioned reasons. Hence, we wish to elucidate how the pandemic affected trauma volumes and injury patterns, as well as discuss lessons learned from our experience. These findings will help to inform how scarce healthcare resources and personnel could be allocated in future pandemic situations.

\section{Methods}

\section{Study cohort}

A retrospective review of the trauma registry was performed of all trauma activations at our American College of Surgeons designated level 1 pediatric trauma center from March 27th through May 14th, 2020. This time interval corresponded with both the peak of the regional pandemic in the surrounding community and was the interval in which COVID-19-related changes to the trauma activation protocols were in place at our institution. During these dates, we merged our three tiers of trauma responses. As with most institutions, traumas at our center are triaged into three levels, with one being the most acute, and three being the least. In the pre-pandemic years, level 1 and two traumas resulted in an automatic trauma activation with the surgery team present during the initial evaluation and resuscitation phase. During the pandemic, only level 1 traumas resulted in a full activation of the trauma team; the activation criteria for which did not change. Traumas which did not meet level 1 criteria were triaged initially by the emergency department team, who would subsequently activate the trauma team if necessary. Additionally, in response to the deluge of adult patients with COVID-19 to centers around the region, our trauma center agreed to accommodate adults with traumatic injuries up to 30 years of age. However, adult patients were excluded from the analysis. Our center returned to a pre-pandemic method of trauma activation and triage on $<<<>>>$ once the intensity of the pandemic subsided regionally.

Data on all trauma activations were compared to the activations which occurred in 2018 and 2019 in the same time interval. Patients who were initially designated as a trauma but on later evaluation were found to have not had a traumatic mechanism were excluded, as were cases with missing data.

\section{Data collection and analysis}

After obtaining institutional review board approval, the trauma registry was queried for characteristics such as age, ethnicity, estimated household income, trauma activation level, Injury Severity Score (ISS), disposition from the emergency room (ER), and final disposition. We further assessed reported physical abuse, if a physical abuse investigation was initiated, and if the patient went home with an alternative caregiver due to physical abuse. These data were compared across the three time periods using the Student's $t$ test, Chi-squared, and Fisher's exact tests where appropriate.

\section{Results}

There were 111, 100, and 52 trauma activations during the study interval in 2018, 2019, and 2020, respectively. There were fewer highest severity level 1 activations in 2020 compared to 2018 and 2019 ( 1 vs 5 and 9; $p=0.006$ ). The median injury severity score (ISS) was higher in 2020 compared to both 2018 and 2019 (5 vs 4 and 4, $p<0.01$ ). There was no difference in the median age of patients $(p=0.33$ ) across 2020 (7-years; IQR 2-11), 2018 (7-years; IQR 3-14), and 2019 (8 years; IQR 3-14). There was a proportional decrease in the number of African American and Asian patients in 2020 compared to prior years $(p<0.01)$. Hispanic patients represented a smaller number of trauma activations during the pandemic compared to the prior year $(p<0.02)$ (Table 1$)$.

Regarding disposition from the emergency room (ER) (Table 2), more patients went directly to the operating room in 2020 (21\%) compared to $2018(8 \%)$ and 2019 (6\%). A smaller proportion of children were able to be discharged home from the ER in 2020 (12\%) compared to 2018 (37\%) and 2019 (33\%). However, these differences did not meet the statistical threshold for significance. The proportion of ICU admissions in 2020 did not appear different compared to prior years.

The injury types and mechanisms are shown in Table 3. There was no difference in the proportion of blunt versus penetrating trauma between years $(p=0.57)$. There were no pedestrians struck by automobiles in 2020 compared to 12 and 14 in 2018 and 2019, respectively. There were a greater proportion of injuries from falls during 2020 compared to prior years.

There did not appear to be an increase in child abuse reports in $2020(N=2 ; 6 \%)$ compared to $2018(N=4 ; 4 \%)$ and $2019(N=9 ; 9 \%)$. Nor were there more child abuse investigations during the pandemic $(N=2 ; 6 \%)$ compared to $2018(N=8 ; 7 \%)$ and $2019(N=9 ; 9 \%)$. 
Table 1 Demographics of patients by year

\begin{tabular}{lcccc}
\hline & $2018, N(\%)$ & $2019, N(\%)$ & $2020, N(\%)$ & $p$ value \\
\hline Age & & & & \\
$\quad$ Median (interquartile range) & $7[3-14]$ & $8(3-14)$ & $7(2-11)$ & 0.33 \\
Race & & & & \\
Caucasian & $44(40)$ & $22(22)$ & $23(44)$ & $<0.01$ \\
African American & $17(15)$ & $17(17)$ & $4(8)$ & \\
Asian & $14(13)$ & $17(17)$ & $2(4)$ & \\
Other & $35(32)$ & $40(40)$ & $20(38)$ & \\
Unspecified & $0(0)$ & $4(4)$ & $3(6)$ & \\
Ethnicity & $9(8)$ & $21(21)$ & $5(10)$ & \\
Hispanic & $102(92)$ & $79(79)$ & $46(90)$ & \\
Non-Hispanic & & & $23(44)$ & \\
Primary Payor & $40(36)$ & $56(56)$ & $27(52)$ & \\
Medicaid & $50(45)$ & $36(36)$ & $2(4)$ & \\
Commercial & $17(15)$ & $8(8)$ & $0(0)$ & \\
Self-Pay & $4(4)$ & $0(0)$ & & \\
Unspecified & & & \\
\hline
\end{tabular}

Table 2 Disposition of patients from the emergency department by year

\begin{tabular}{lcccc}
\hline & $2018, N(\%)$ & $2019, N(\%)$ & $2020, N(\%)$ & $p$ value \\
\hline Home & $41(37)$ & $32(33)$ & $4(12)$ & 0.16 \\
Floor & $52(46)$ & $48(49)$ & $19(58)$ & \\
Intensive Care & $7(6)$ & $9(9)$ & $2(6)$ & \\
$\quad$ Unit & & & & \\
Operating Room & $9(8)$ & $6(6)$ & $7(21)$ & \\
Transfer & $1(1)$ & $0(0)$ & $0(0)$ & \\
Unknown & $2(2)$ & $3(3)$ & $1(3)$ & \\
\hline
\end{tabular}

\section{Discussion}

The COVID-19 pandemic placed immense pressure on healthcare systems with shortages of PPE and personnel [6]. The care and management of trauma patients is resource intensive in both respects. Our health system alone treated more than 5700 inpatients with COVID-19 during the early stages of the pandemic [7]. Due to such overwhelming volumes, there was diversion of pediatric resources in terms of both personnel and beds to increase capacity for adult COVID-19 patients [8, 9].

Measures put in place by governments, combined with public fears of COVID-19 transmission resulted in large swaths of the population staying at home after the closure of schools and non-essential businesses. Countries that had already experienced the peaks of their own COVID-19 outbreaks and had already implemented similar mitigations strategies showed a decrease in overall trauma volumes $[10,11]$.
Table 3 Injury types and mechanisms by year

\begin{tabular}{lccc}
\hline & $2018, N(\%)$ & $2019, N(\%)$ & $2020, N(\%)$ \\
\hline Blunt & & & \\
MVC & $11(10)$ & $4(4)$ & $3(6)$ \\
Autoped & $12(11)$ & $14(14)$ & $0(0)$ \\
Fall & $59(53)$ & $56(56)$ & $38(73)$ \\
Assault & $3(3)$ & $1(1)$ & $3(6)$ \\
Collision & $7(6)$ & $11(11)$ & $2(4)$ \\
Self-injury & $1(1)$ & $1(1)$ & $0(0)$ \\
Struck by object & $5(5)$ & $0(0)$ & $0(0)$ \\
Other & $6(5)$ & $6(6)$ & $0(0)$ \\
Total blunt & $104(94)$ & $93(93)$ & $46(88)$ \\
Penetrating & & & \\
Dog bite & $3(3)$ & $1(1)$ & $3(6)$ \\
Stab & $2(2)$ & $3(3)$ & $1(2)$ \\
Laceration & $0(0)$ & $3(0)$ & $1(2)$ \\
GSW & $2(2)$ & $0(0)$ & $1(2)$ \\
Total penetrating & $7(6)$ & $7(7)$ & $6(12)$ \\
\hline
\end{tabular}

$M V C$ motor vehicle collision, Autoped pedestrian struck by automobile, GSW gunshot wound

Additionally, as the pandemic progressed, it became apparent from the published literature that while the prevalence of COVID-19 in the pediatric population was less than that of the adult population, children could serve as asymptomatic carriers of the virus [12]. Due to this concern, and the anticipated decrease in trauma volume, the way trauma was initially evaluated was modified. This was done to conserve limited supplies of PPE, as well as to minimize potential reciprocal exposure between patients and hospital staff as the staffing and pace of a 
trauma evaluation often precluded ideal infection control practices [13-16]. We noted a significant decrease in total trauma activations of almost $50 \%$ compared to the preceding 2 years during the peak of the pandemic, which is consistent with the previously published literature [17].

We saw small but significantly increased ISS score from 2020 compared to prior years. When looking solely level 1 activations, where there was no change in activation criteria, there was only a single level 1 activation, which was significantly fewer than the same period in the two preceding years. Overall trauma activation patients were more severely injured; however, the number of highest severity patients was decreased. Presumably, this could be a result of a general reluctance of the public to present to healthcare facilities. It could also be a byproduct of the modified triage process, where only after initial triage by the emergency department would appropriate cases result in a trauma team activation, resulting in selection of more seriously injured patients for trauma activation.

Another notable secondary effect of the pandemic was a sharp decline in traffic and traffic-related injuries [18, 19], which was consistent with our data which showed no automobile versus pedestrian traumas during the peak of the pandemic. This is presumably due to the stay-at-home orders combined with the high rate of compliance in our region during the peak of the pandemic (Czeisler, Mark et al. "COVID-19: public compliance with and public support for stay-at-home mitigation strategies" medRxiv (2020)). At the same time, there was an increase in the proportion of patients who were evaluated for falls. This could be secondary to the halt in in-person instruction at schools and the shift to children being at home. We also noted that the absolute number of falls was less than nonpandemic years, thus the increased proportion of falls may be due to the decrease in motor vehicle-related trauma as a share of trauma volume. The increase in patients who went directly to the operating room from the emergency room is the result of COVID-19 precautions. Patients requiring urgent but not emergent operations were held in the emergency room while waiting for the results of a rapid COVID-19 test.

The proportion of minority trauma patients was decreased compared to non-pandemic years. Minority populations have been shown to be more likely to be hospitalized and were more likely to develop severe disease [20,21]. Whether minority populations were more adherent to social isolation mandates and stay-at-home orders or were less likely to seek care if injured is unknown and may benefit from further study. We also noted a relatively higher proportion of Caucasian patients as well as self-pay patients in 2018 compared to 2019 and 2020. Whether this is a result of changes in hospital system and catchment, data collection, annual variation, or some other unknown confounder is unclear.
Based on data from previous disasters, another postulated effect of the emotional trauma, economic hardship, and stay-at-home orders was that there would be an increased in domestic violence and self-harm [22, 23]. This trend was not appreciated in this study, as we did not see any significant increase in child abuse reports or investigations, nor did we note an increase in cases of self-injury. However, whether this is secondary to underreporting as a result of children being sequestered at home is unclear as educational professionals and medical personnel account for over $30 \%$ of child abuse reporting [24]. Additional investigations may be needed to truly determine if there were any changes in the incidence of child abuse because of the pandemic.

Lessons taken from this experience may not be universally applicable in all contexts. This review was conducted at a tertiary children's hospital with an independent physical plant, staff, and leadership structure. As with many children's hospitals around the country, our center is integrated into a much larger hospital system and physically adjoins a large flagship adult hospital. This allowed a low friction transfer of resources to other parts of the campus and hospital system. Even in cases where a children's hospital is not part of a larger system or physically connected to another center, it could still function as a part of a wider part of a multi-institutional or government-led response in a pandemic situation. While, a future pandemic may not have identical characteristics or courses, for example a unique quality of COVID-19 was its relatively benign course in the younger pediatric population (REF). It would be reasonable to assume that management of future pandemic situations would involve some variation of a stay-at-home order and a corresponding decrease in trauma volumes. Trauma resources may still be able to be diverted to areas of greater need, such as the transfer of personnel to pandemic-related care or increased pediatric ward capacity to allow the concentration of regional pediatric care to a single center.

These data suggest that the measured reduction of trauma care resources in such a pandemic situation is appropriate, which would allow surgical, emergency room, and critical care resources to be reallocated to areas of greater demand. However, further review of COVID-19's impact on pediatric trauma is needed to allow hospitals and trauma systems plan appropriately as they face future potential pandemic situations.

Author contributions YS: study design, data acquisition, analysis, interpretation of data, and drafting and revision of the manuscript. CK: study design, data acquisition, analysis, interpretation of data, and drafting and revision of the manuscript. SK: data acquisition and drafting and revision of the manuscript. SJ: data acquisition and drafting and revision of the manuscript. DS: data acquisition and drafting and revision of the manuscript. JP: study design, data acquisition, analysis, interpretation of data, and drafting and revision of the manuscript. 
CS: study design, data acquisition, analysis, interpretation of data, and drafting and revision of the manuscript.

Funding None.

\section{Declarations}

Conflict of interest The authors declare that they have no competing interest.

\section{References}

1. Dong E, Du H, Gardner L (2020) An interactive web-based dashboard to track COVID-19 in real time. Lancet Infect Dis 20(5):533-534

2. Zhu N, Zhang D, Wang W, Li X, Yang B, Song J, Zhao X, Huang B, Shi W, Lu R et al (2020) A novel coronavirus from patients with pneumonia in China, 2019. N Engl J Med. https://doi.org/10. 1056/NEJMoa2001017. Cited 31 Aug 2020

3. Kvasnovsky CL, Shi Y, Rich BS, Glick R, Soffer S, Lipskar AM, Dolgin S, Bagrodia N, Hong A, Prince JM et al (2020) Limiting operations for acute appendicitis in children: lessons learned from the U.S. epicenter of the COVID-19 pandemic. J Pediatr Surg. https://www.ncbi.nlm.nih.gov/pmc/articles/PMC7309720/. Cited 30 Aug 2020

4. Hsieh T, Dedhia RD, Chiao W, Dresner H, Barta RJ, LyfordPike S, Hamlar D, Stephan SJ, Schubert W, Hilger PA (2020) A guide to facial trauma triage and precautions in the COVID-19 pandemic. Facial Plast Surg Aesthetic Med 22(3):164-169

5. Ashford RU, Nichols JS, Mangwani J (2020) Annotation: the COVID-19 pandemic and clinical orthopaedic and trauma surgery. J Clin Orthop Trauma 11(3):504-505

6. Ramachandran P, Swamy L, Kaul V, Agrawal A, Narasimhan M (2020) Powerless in the ICU. Chest. https://www.ncbi.nlm.nih. gov/pmc/articles/PMC7245235/. Cited 2 Sep 2020

7. Richardson S, Hirsch JS, Narasimhan M, Crawford JM, McGinn T, Davidson KW, Barnaby DP, Becker LB, Chelico JD, Cohen SL et al (2020) Presenting characteristics, comorbidities, and outcomes among 5700 patients hospitalized with COVID-19 in the New York City Area. JAMA 323(20):2052-2059

8. Petrone B, Iturriaga C, Mauri T, Sgaglione N (2020) Impact of Covid-19 pandemic on orthopaedics at Northwell Health, New York. Arthrosc Sports Med Rehabil. https://www.ncbi.nlm.nih. gov/pmc/articles/PMC7245196/. Cited 2 Sept 2020

9. DePeralta DK, Hong AR, Choy C, Wang J, Ricci JP, MarcanoBenfante BV, Lipskar AM (2020) Primer for intensive care unit (ICU) redeployment of the noncritical care surgeon: Insights from the epicenter of the coronavirus disease 2019 (COVID-19) pandemic. Surgery 168(2):215-217

10. Pellegrini M, Roda M, Di Geronimo N, Lupardi E, Giannaccare G, Schiavi C (2020) Changing trends of ocular trauma in the time of COVID-19 pandemic. Eye 34(7):1248-1250

11. Camine T, Rembisz A, Barron R, Baldwin C, Kromer M (2020) Decrease in trauma admissions with COVID-19 pandemic. West J Emerg Med 21(4). https://escholarship.org/uc/item/05n535hg. Cited 2 Aug 2020
12. Kelvin AA, Halperin S (2020) COVID-19 in children: the link in the transmission chain. Lancet Infect Dis 20(6):633-634

13. Samsami M, Zebarjadi Bagherpour J, Nematihonar B, Tahmasbi H (2020) COVID-19 pneumonia in asymptomatic trauma patients; report of 8 cases. Arch Acad Emerg Med. 8(1). https://www.ncbi. nlm.nih.gov/pmc/articles/PMC7158871/. Cited 1 Sep 2020

14. Lai C-C, Liu YH, Wang C-Y, Wang Y-H, Hsueh S-C, Yen M-Y, Ko W-C, Hsueh P-R (2020) Asymptomatic carrier state, acute respiratory disease, and pneumonia due to severe acute respiratory syndrome coronavirus 2 (SARS-CoV-2): Facts and myths. J Microbiol Immunol Infect 53(3):404-412

15. De Vitis R, Passiatore M, Perna A, Proietti L, Taccardo G (2020) COVID-19 contagion and contamination through hands of trauma patients: what risks and what precautions? J Hosp Infect 105(2):354-355

16. Dutton RP, Grissom TE, Herbstreit F (2020) COVID-19 and trauma care: improvise, adapt and overcome. Anesth Analg. https://journals.lww.com/https://doi.org/10.1213/ANE.00000 00000004944. Cited 27 Aug 2020. (Publish Ahead of Print)

17. Nuñez JH, Sallent A, Lakhani K, Guerra-Farfan E, Vidal N, Ekhtiari S, Minguell J (2020) Impact of the COVID-19 pandemic on an emergency traumatology service: experience at a tertiary trauma centre in Spain. Injury 51(7):1414-1418

18. Sutherland M, McKenney M, Elkbuli A (2020) Vehicle related injury patterns during the COVID-19 pandemic: what has changed? Am J Emerg Med 38(9):1710-1714

19. Chauhan A, Singh RP (2020) Decline in PM25 concentrations over major cities around the world associated with COVID-19. Environ Res 187:109634

20. Raisi-Estabragh Z, McCracken C, Bethell MS, Cooper J, Cooper C, Caulfield MJ, Munroe PB, Harvey NC, Petersen SE (2020) Greater risk of severe COVID-19 in Black, Asian and Minority Ethnic populations is not explained by cardiometabolic, socioeconomic or behavioural factors, or by $25(\mathrm{OH})$-vitamin $\mathrm{D}$ status: study of 1326 cases from the UK Biobank. J Public Health Oxf Engl. https://www.ncbi.nlm.nih.gov/pmc/articles/PMC7449237/. Cited 4 Oct 2020

21. Price-Haywood EG, Burton J, Fort D, Seoane L (2020) Hospitalization and mortality among black patients and white patients with Covid-19. N Engl J Med. https://www.ncbi.nlm.nih.gov/pmc/artic les/PMC7269015/. Cited 4 Oct 2020

22. Olding J, Zisman S, Olding C, Fan K (2020) Penetrating trauma during a global pandemic: changing patterns in interpersonal violence, self-harm and domestic violence in the Covid-19 outbreak. The Surgeon. https://www.ncbi.nlm.nih.gov/pmc/articles/PMC73 92113/. Cited 2 Sep 2020

23. Anurudran A, Yared L, Comrie C, Harrison K, Burke T (2020) Domestic violence amid COVID-19. Int J Gynecol Obstet 150(2):255-256

24. Child Welfare Information Gateway (2020) Child maltreatment 2018: Summary of key findings. Washington, DC: U.S. Department of Health and Human Services, Administration for Children and Families, Children's Bureau

Publisher's Note Springer Nature remains neutral with regard to jurisdictional claims in published maps and institutional affiliations. 


\section{Authors and Affiliations}

\section{Yan Shi ${ }^{1,2}$. Charlotte Kvasnovsky ${ }^{1,2}$. Saira Khan ${ }^{3} \cdot$ Shelley Jain $^{3,4}$. Danielle Sargeant ${ }^{5} \cdot$ Abdulraouf Lamoshi $^{1,2}$. Jose Prince ${ }^{1,2}$. Chethan Sathya ${ }^{1,2}$}

Charlotte Kvasnovsky

ckvasnovsky@northwell.edu

Saira Khan

skhan61@ pride.hofstra.edu

Shelley Jain

sjain19@northwell.edu

Danielle Sargeant

dmeyerowit@northwell.edu

Abdulraouf Lamoshi

jprince@northwell.edu

Jose Prince

jprince@northwell.edu

Chethan Sathya

csathya@northwell.edu
1 Division of Pediatric Surgery, Cohen Children's Medical Center, 1111 Marcus Avenue, Suite M15, New Hyde Park, NY 11042, USA

2 Department of Surgery, Zucker School of Medicine at Hofstra/Northwell, Hempstead, NY, USA

3 Zucker School of Medicine at Hofstra/Northwell, Hempstead, NY, USA

4 Department of Surgery, Northwell Health, Lake Success, NY, USA

5 Division of Pediatric Surgery, Gambino Pediatric Trauma and Imaging Center, Cohen Children's Medical Center, New Hyde Park, NY, USA 